\title{
Effect of Copper Content, Initial Structure, and Scheme of Treatment on Magnetic Properties of Ultra-Thin Grain Oriented Electrical Steel
}

\author{
M. L. Lobanov ${ }^{a}$, G. M. Rusakov ${ }^{a, b}$, and A. A. Redikul'tsev ${ }^{a}$ \\ ${ }^{a}$ Yeltsin Ural Federal University, pr. Mira 19, Ekaterinburg, 620002 Russia \\ ${ }^{b}$ Institute of Metal Physics, Ural Branch, Russian Academy of Sciences, \\ ul. S. Kovalevskoi 18, Ekaterinburg, 620990 Russia \\ e-mail: redikultsev@mail.ru \\ Received April 5, 2012
}

\begin{abstract}
The effect of the copper content, initial structure, and scheme of treatment on the magnetic properties of an ultra-thin grain oriented electrical steel has been investigated. In the material with copper and an initial sharp texture, the nucleation of new grains upon primary recrystallization is connected with deformation twins; in the samples without copper and with copper and diffuse texture, it is connected predominantly with shear bands and transition bands. Upon heating at a rate of $\sim 0.004 \mathrm{~K} / \mathrm{s}$, the temperature of primary recrystallization in the copper-bearing samples is considerably higher than in the copper-free material. Upon heating at a rate of $\sim 4 \mathrm{~K} / \mathrm{s}$ the appearance of new grains occurs almost simultaneously for all of the studied samples. In the samples with copper and initial sharp texture after annealing at $1050^{\circ} \mathrm{C}$, a significant part of the volume is occupied by grains that had undergone normal grain growth; in the samples without copper and with copper and diffuse texture, anomalous growth is hardly observed at all. To obtain high final magnetic properties of the ultra-thin grain oriented electrical steel produced by the Littmann method, it has been suggested to use an grain oriented electrical steel with $0.5 \% \mathrm{Cu}$ that exhibits the diffuse orientation of grains as the workpiece.
\end{abstract}

Keywords: ultra-thin grain oriented electrical steel, grain orientation, primary recrystallization, normal grain growth (secondary recrystallization), magnetic properties

DOI: $10.1134 / \mathrm{S} 0031918 X 13070077$

\section{INTRODUCTION}

The ultra-thin $(0.01-0.15 \mathrm{~mm})$ grain oriented electrical steel is the most important soft-magnetic material employed for fabricating magnetic circuits of high-frequency devices. The technology of its production involves cold rolling of strips of a coarse-grained $(5-30 \mathrm{~mm})$ grain oriented electrical steel (GOES) with a perfect cube-on-edge (Goss) texture $\sim 0.3 \mathrm{~mm}$ (or smaller) thick and subsequent annealing for primary recrystallization [1]. To date appreciable interest in the mechanism of the formation of the texture that determines the unique properties of this product is retained [2-4].

The workpiece (thin GOES) with a certain grain size and the minimum copper content is conventionally used to produce the ultra-thin GO electrical steel. This work is devoted to studying the effect of the copper content, initial structure, and annealing parameters on the magnetic properties of this product.

\section{EXPERIMENTAL}

Three series of samples that consist of sets of GOES strips with dimensions of $0.30 \times 280 \times 30 \mathrm{~mm}$ were used as the materials for the investigation. The samples differed in chemical composition (mainly the presence of copper), the perfection of the Goss texture (110)[001] (magnetic properties), and the average size of grains that underwent secondary recrystallization (see Table 1 and Fig. 1). For each series, samples with about the same level of magnetic inductions $B_{800}$ and $B_{2500}$ were selected. The deviation of grains that underwent secondary rectystallization from the ideal orientation (110)[001] is expressed by the angle $\alpha$. The angle $\alpha$ is the angle between the rolling direction (RD) and the projection of a crystallographic direction [001] onto the rolling plane (the latter is specified by the normal direction (ND) to it). The angle $\alpha$ can be determined from direct pole figures or from the direction of main domains [5].

To obtain samples of the ultra-thin GOES $(0.1 \mathrm{~mm})$, two schemes of treatment were employed (Fig. 2). All samples were rolled to a final thickness of $0.1 \mathrm{~mm}$ (degree of reduction 67\%). Then, half of the samples were subjected to annealing for primary recrystallization (PR) from 300 to $850^{\circ} \mathrm{C}$ with a heating rate of $0.004 \mathrm{~K} / \mathrm{s}(15 \mathrm{~K} / \mathrm{h}$, so-called controllable heating) (schematic 1, Fig. 2a). The second half of the 
Table 1. Chemical composition, magnetic properties, and average angle $\alpha$

\begin{tabular}{c|c|c|c|c|c|c|c|c|c|c}
\hline \multirow{2}{*}{$\begin{array}{c}\text { No. of se- } \\
\text { ries of } \\
\text { samples }\end{array}$} & \multicolumn{7}{|c|}{ Content of elements, wt \% } & \multicolumn{2}{c|}{ Min-max/average } & \multirow{2}{*}{$\alpha, \mathrm{deg}$} \\
\cline { 2 - 8 } 1 & 0.002 & 3.12 & 0.28 & 0.003 & 0.008 & 0.002 & 0.53 & $\frac{1.87-1.93}{\mathbf{1 . 9 0}}$ & $\frac{1.94-1.99}{\mathbf{1 . 9 7}}$ & 6 \\
2 & 0.002 & 3.12 & 0.28 & 0.003 & 0.008 & 0.002 & 0.53 & $\frac{1.67-1.76}{\mathbf{1 . 7 3}}$ & $\frac{1.79-1.87}{\mathbf{1 . 8 4}}$ & 13 \\
3 & 0.002 & 3.10 & 0.24 & 0.004 & 0.007 & 0.002 & 0.05 & $\frac{1.79-1.85}{\mathbf{1 . 8 0}}$ & $\frac{1.89-1.94}{\mathbf{1 . 9 0}}$ & 10 \\
\hline
\end{tabular}

samples was annealed for PR via placement into a furnace heated to $850^{\circ} \mathrm{C}$ for $5 \mathrm{~min}$ (so-called recrystallization annealing) (schematic 2, Fig. 2b). For both schemes, the refining high-temperature treatment (HTT) at $1050^{\circ} \mathrm{C}$ in an atmosphere of dry hydrogen was used for final annealing. After each stage of treatment, the magnetic properties of the samples were measured.

The domain structure of the samples was investigated using an ORB magnetic lens by the method of magnetic metallography. Their magnetic properties were measured on a TWM-8S single-band device. The magnetic induction was determined at fixed values ( 800 and $2500 \mathrm{~A} / \mathrm{m}$ ) of the magnetic-field strength. Specific magnetic losses were determined at a frequency of the electromagnetic field of $50 \mathrm{~Hz}$ (measurements on the TWM-8S single-band device at frequencies that greatly exceed $50 \mathrm{~Hz}$ are impossible) and magnetic inductions 1.5 and $1.7 \mathrm{~T}\left(P_{1.5 / 50}\right.$ and $P_{1.7 / 50}$, respectively). The measuring errors are as follows: $\pm 0.5 \% \pm 2$ units of the last digit place for magnetic induction $B, \pm 1.0 \% \pm 2$ units of the last digit place for specific losses $P$. All the obtained data were averaged over ten measurements. The microstructure was studied on a JEOL JCM6490LV electron microscope equipped with an Oxford Instruments attachment for electron backscatter diffraction. In the course of the experiment, the relationship between the initial magnetic properties and structure of the samples and the obtained properties of the ultra-thin GOES was investigated.

As the laboratory system, we adopted the coordinate system, the axes of which are connected with RD of cold rolling, ND to the rolling plane, and perpendicular direction (TD) to the above directions, so that all the three directions form the right-hand triple of vectors.

\section{STRUCTURAL TRANSFORMATIONS AND MAGNETIC PROPERTIES OF SAMPLES}

The conducted measurements of the properties of samples after intermediate and final heat treatments showed substantial distinctions in changes of the magnetic induction depending on the degree of perfection of the initial texture, the presence of copper in the
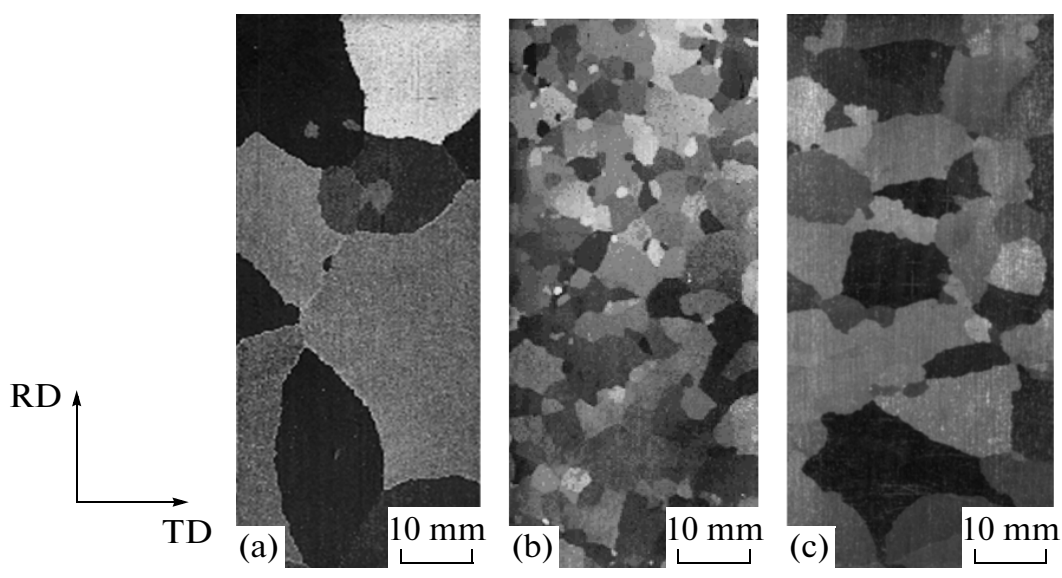

Fig. 1. Macrostructure of initial samples of the GOES: (a) series 1, (b) series 2, and (c) series 3. 


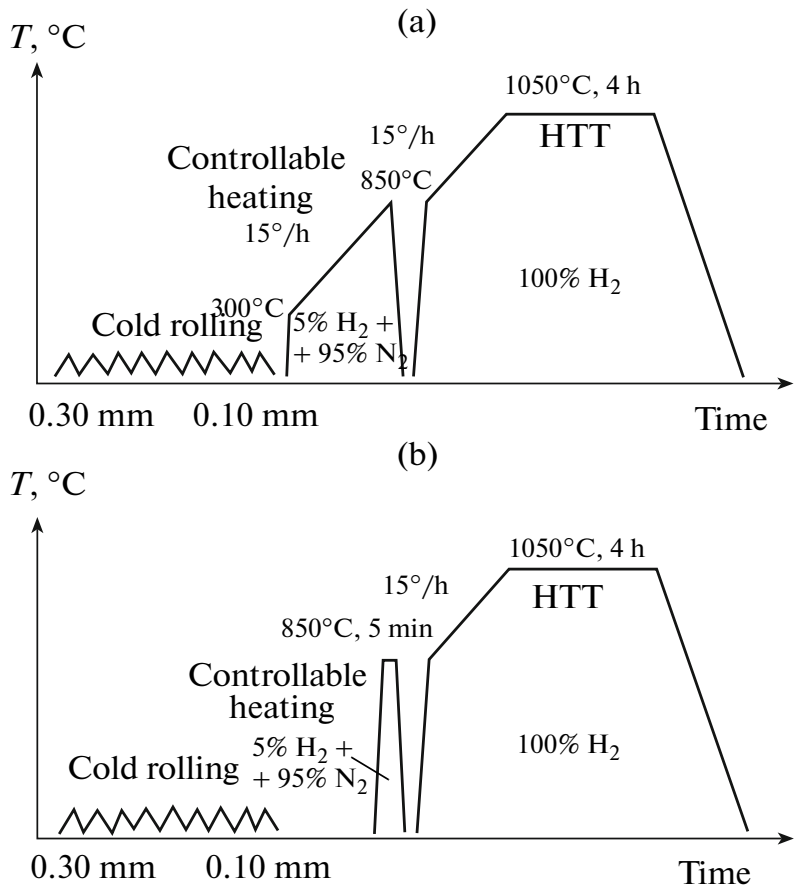

Fig. 2. Schemes of production of ultra-thin GOES (see text for explanation).

material, and the heating rate upon annealing for PR (Fig. 3, Table 2).

After both types of annealing magnetic properties of samples of all the series prove, on the average, to be appreciably lower than their initial properties. However, this difference is smaller in series 2 (samples with the lowest level of the initial magnetic induction) and, in this series, there show up single samples in which the reproduction of magnetic properties is observed after rolling and slow annealing for PR. It should also be noted that, for all heating rates upon annealing for $\mathrm{PR}$, a clearly pronounced correlation dependence is observed only in this series; the higher the initial magnetic properties, the higher the magnetic properties of the material are after intermediate annealing (Fig. 3).

HTT of samples of series 2 leads to the marked improvement of magnetic properties, regardless of the type of the preceding treatment (Fig. 3). For samples of series 1 , HTT results in a small reduction in the magnetic properties. As for samples of series 3, after the completion of the cycle of heat treatments, their magnetic properties become somewhat better in the case of rapid heating for PR and remain almost unaltered in the case of annealing for PR at a slow rate.

Investigations of the macrostructure of samples after HTT showed that, in the material with an initial sharp texture containing $\sim 0.5 \mathrm{wt} \%$ copper, regardless of the parameters of annealing for PR, a greater part of the volume is occupied by grains undergone secondary recrystallization. The structure resulting from HTT is (a)

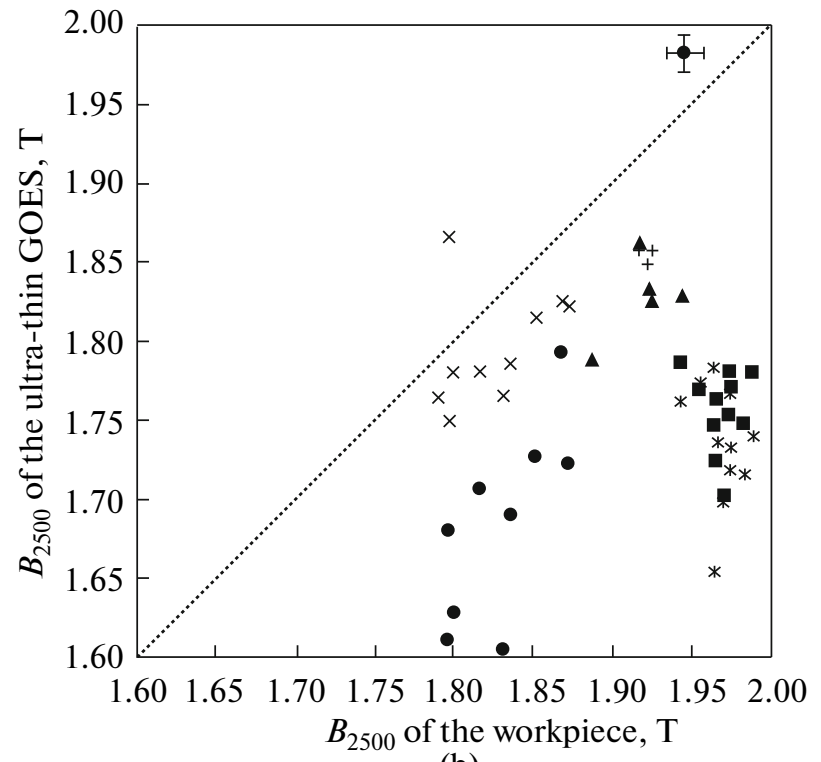

(b)

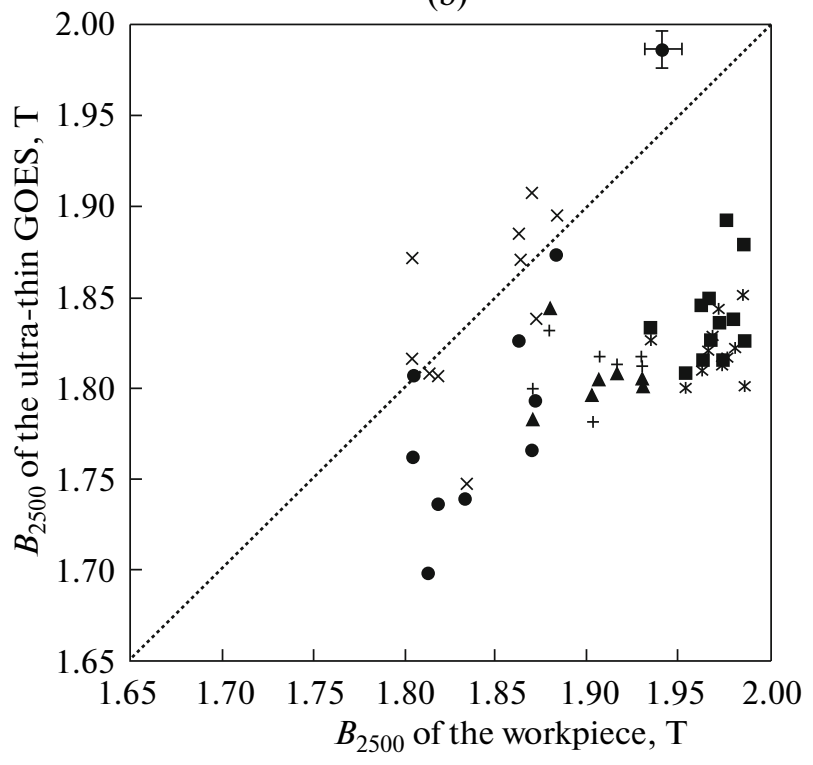

Fig. 3. Interrelation between the initial magnetic induction of the workpiece and the induction of the ultra-thin GOES: (a) annealing for PR at $850^{\circ} \mathrm{C}$ for $5 \mathrm{~min}$ and (b) annealing for PR with a heating rate of $\sim 0.004 \mathrm{~K} / \mathrm{s} ; \mathbf{\square}, \bullet$, $\Delta$ after PR; $*, \times,+$ after HTT; $\mathbf{n}, *$ samples of series 1 ; $\boldsymbol{\bullet}, \times$ samples of series 2 ; and $\mathbf{\Lambda},+$, samples of series 3 .

an aggregate of macroregions replicating (with allowance for deformation) the coarse-grained structure of initial samples after secondary recrystallization. In this case, a greater part of the former cube-on-edge crystals is completely occupied by new anomalously grown grains. In the two remaining series of samples, regardless of the used scheme of treatment, anomalously grown crystallites are very rarely observed as individual grains.

The best magnetic properties $\left(B_{2500} \sim 1.85 \mathrm{~T}\right)$ are fixed for samples of series 2 subjected to slow heating 
Table 2. Interrelation of initial magnetic properties with properties of the ultra-thin GOES and its macrostructure after different treatments

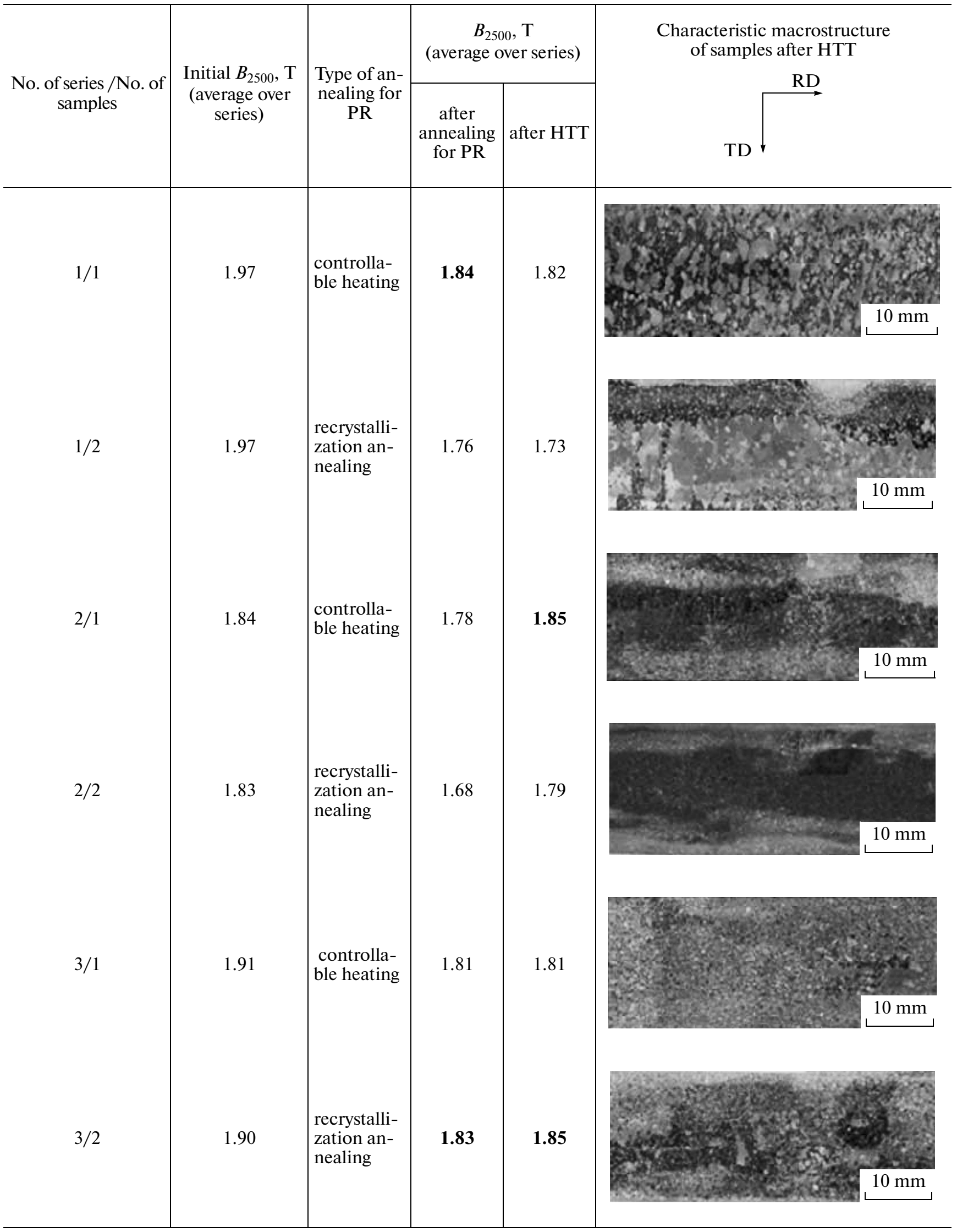



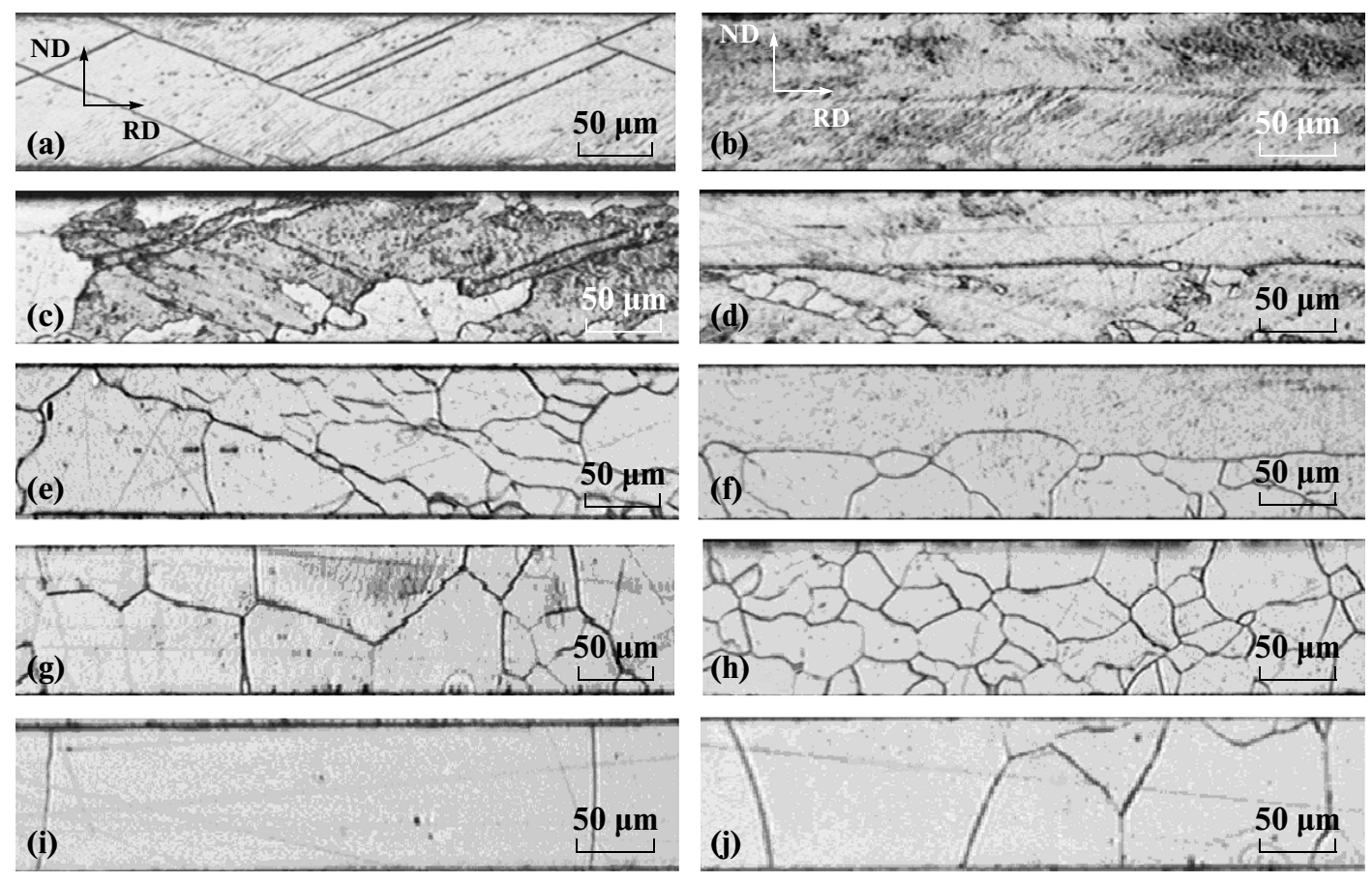

Fig. 4. Structure of single crystals of the $\mathrm{Fe}-3 \% \mathrm{Si}-0.5 \% \mathrm{Cu}$ alloy after annealing with different rates: left-hand column: initial sharp texture (110)[001], heating rate $\sim 0.004 \mathrm{~K} / \mathrm{s}$; right-hand column: initial diffuse texture (110)[001], heating rate $\sim 4 \mathrm{~K} / \mathrm{s}$; (a, b) $300^{\circ} \mathrm{C}$; (c, d) $650^{\circ} \mathrm{C}$; (e, f) $750^{\circ} \mathrm{C}$; (g, h) $850^{\circ} \mathrm{C}$; and $(\mathrm{i}, \mathrm{j}) 950^{\circ} \mathrm{C}$.

for PR and HTT and for samples of series 3 after annealing for PR with high rate and HTT.

To explain the observed regularities of the formation of magnetic properties of samples, evolution of the structure of materials of all the series in the PR process was investigated metallographically. The investigation was performed using gradient annealings in a temperature range of $300-1000^{\circ} \mathrm{C}$. Upon the modeling of annealing with slow heating, samples were placed into a cold furnace in the zone of the temperature gradient, heated to $300^{\circ} \mathrm{C}$ together with the furnace (heating rate $\sim 0.1 \mathrm{~K} / \mathrm{s}$ ), then heated to $900^{\circ} \mathrm{C}$ with a rate of $0.004 \mathrm{~K} / \mathrm{s}$; subsequently, cooling in air was performed. Upon the modeling of annealing with rapid heating, samples were placed into the heated furnace in the zone of temperature gradient, where they were held for $5 \mathrm{~min}$ with subsequent cooling in air. The microstructure of samples is shown in Fig. 4.

In samples of series 1 (high copper content, a sharper initial texture), in the overwhelming majority of grains in the deformed state, numerous deformation twins were observed (Fig. 4a). The structure of grains in the copper-bearing material with the initial diffuse texture (series 2), as in the copper-free material (series 3) in the deformed state, was mainly characterized by the presence of shear bands; some crystallites proved to be broken down into several deformation bands separated by transition bands (Fig. 4b). In the material with an initial sharp texture, regardless of the heating rate, the nucleation of new grains upon PR is connected with deformation twins (Fig. 4c); in samples of the other two series, it is predominantly connected with shear bands and transition bands (Fig. 4d).

In samples with copper (series 1 and 2), upon slow heating, the appearance of the first PR nuclei occurred at a higher temperature $\left(\sim 630^{\circ} \mathrm{C}\right.$ compared to $\sim 550^{\circ} \mathrm{C}$ ) in the material of series 3 . In samples of series 3 , at $\sim 660^{\circ} \mathrm{C}$, the PR process was almost complete, and the structure consisted of fairly equiaxed grains. In samples of series 1 and 2, at this temperature, the development of PR was observed with the occupation of about $50 \%$ of the material volume by new grains (Fig. 4f). The total lack of deformed regions in the structure of copper-bearing samples was observed only at a temperature of $\sim 750^{\circ} \mathrm{C}$ (Fig. $4 \mathrm{~g}$ ). The larger size of grains after the completion of PR in copper-bearing samples as compared to samples of series 3 indicates that the number of nuclei of new defect-free crystallites is noticeably smaller. The mechanism of the analogous effect of copper on the structure upon deformation and recrystallization of samples remains unsolved.

Regardless of the copper content in alloys, new grains in samples subjected to rapid heating for PR arose almost simultaneously at a temperature of $\sim 750^{\circ} \mathrm{C}$. At a temperature of $\sim 880^{\circ} \mathrm{C}$, the $\mathrm{PR}$ proved to be complete in all samples. In this case, the grain size in samples subjected to rapid heating for PR turned out to be fairly close for the both chemical compositions of alloys, and this grain size is substantially smaller than in samples heated slowly to $850^{\circ} \mathrm{C}$. 
The further heating of samples of series 1, regardless of the type of annealing for PR, was accompanied by the appearance in the structure of single grains that underwent secondary recrystallization beginning at a temperature of $\sim 950^{\circ} \mathrm{C}$ (Fig. 4i). In the structure, upon heating samples of series 2 and 3 in the HTT temperature range, an increase in the average grain size to a size that coincides with the sample thickness was observed for the both cases of the preceding heat treatment; i.e., normal grain growth was effected (Fig. 4j).

\section{DISCUSSION OF RESULTS}

The results obtained in this work concerning changes in the magnetic properties of samples can be explained in terms of the processes of the texture formation upon deformation and subsequent recrystallizations. Upon the deformation of the initial cube-onedge single crystal, the main part of the crystal lattice transforms into octahedral orientations $\{111\}\langle h h l\rangle$. Upon further heating for PR, new Goss grains grow from regions containing mesostructure elements (twins, transition bands, shear bands). After the completion of PR, the Goss orientation dominates in the material $[4,6,7]$. Based on the previous investigations [7], it can be assumed that nuclei that arise at deformation twins possess the most perfect Goss orientation. It should be also noted that, upon PR, it is these nuclei that appear in the first place [8].

The heating rate of the deformed metal upon annealing exerts an influence on the mechanisms of grain nucleation and, hence, on the PR texture. If heating occurs rapidly, each mechanism of grain nucleation has no priority; i.e., the process of grain nucleation takes place using all possible mechanisms and the sharpness of the final texture proves to be worse. If heating occurs slowly, the mechanisms of the nucleation of new grains are separated in time; i.e., it can be assumed that nuclei at twins are formed first and nucleation in shear bands and/or transition bands occurs only in the final stages at higher temperatures. Thus, the texture turns out to be sharper when the series of samples in which the metal is prone to deformation twinning was subjected to slower heating for PR.

Further heating of the metal after PR in which the sharp single-component texture was formed is accompanied by the secondary recrystallization process. In this case, grains that grow via the anomalous mechanism differ in orientation from the matrix texture, i.e., substantially change the texture formed upon PR and, hence, worsen the magnetic properties. It should also be noted that the greater the perfection of the texture formed upon PR, the more probable (at lower temperatures with a higher rate) the occurrence of secondary recrystallization process in this structure.

Samples of series 1 were characterized by a deformed structure containing numerous twins. Correspondingly, following PR, the nucleation mecha- nism predominantly operates, which ensures the maximum perfection of the Goss texture after the completion of PR. It is natural that, in the case of slow heating, the Goss texture proved to be more perfect (a higher magnetic induction, Table 2). Further hightemperature heating resulted in development of the process of anomalous grain growth with the appearance of non-Goss components of the texture, which led to a reduction in the magnetic properties.

Deformation of samples of series 3 and, in particular, series 2 improves the matrix texture. This occurs due to the transformation of the poor goss orientation into more perfect octahedral orientations [4]. In the process of subsequent annealing, following PR, mainly nucleation mechanisms operate, which are connected predominantly with shear bands and transition bands. After the completion of PR, the Goss texture is formed, which is more diffuse compared to samples obtained from the material with the initial perfect orientation. However, this material is characterized by a more disperse domain structure and, importantly, a significantly smaller tendency to anomalous grain growth in it at elevated temperatures. The latter factor makes it possible to substantially improve the magnetic structure of samples upon normal grain growth in the process of high-temperature annealing at the expense of the retention (or possibly improvement) of the Goss orientation of the main part of grains (Table 2).

\section{Optimization of the Technology of Producing Ultra-thin GOES}

The thin copper-free GOES is conventionally used as the workpiece for fabricating the ultra-thin GOES. This is connected with the large grain size, enhanced tendency to deformation twinning, and subsequent fracture of a copper-containing $\mathrm{Fe}-3 \% \mathrm{Si}$ alloy in the process of cold rolling. To obtain final properties in the ultra-thin GOES, the high-temperature annealing in a bell-type furnace in an atmosphere of pure hydrogen can be employed; this annealing allows one to perform deep refining of the material to reduce its specific magnetic losses. In addition, the deposition of the thermostable $\mathrm{MgO}$-based coating on the strip before HTT in combination with the deposition of the insulating aluminum phosphate-based coating on the strip after HTT makes it possible to form a magnetoactive coating on the surface of the material; this coating likewise favors a decrease in the specific magnetic losses and possesses high dielectric properties. However, the performance of HTT involves limitation of the refining temperature because of the possibility of the occurrence of secondary rescrystallization with appreciable worsening of magnetic properties.

These investigations show that the $\mathrm{Fe}-3 \% \mathrm{Si}-$ $0.5 \% \mathrm{Cu}$ alloy (typical thin GOES) with the initial diffuse Goss texture (low-quality steel) can be used as the workpiece for fabricating the ultra-thin GOES. This 
texture markedly decreases the tendency toward deformation twinning and, hence, toward fracture upon cold rolling. The initial diffuse texture of GOES likewise reduces the probability of the occurrence of anomalous grain growth upon HTT, and the presence of copper significantly raises the temperature of the secondary recrystallization start. This allows one to perform the refining treatment of the material at the highest temperatures and to ensure the best magnetic properties (without degradation of the properties due to secondary recrystallization).

\section{CONCLUSIONS}

In this work, the effect of the copper content, initial structure, and scheme of treatment on the magnetic properties of the ultra-thin GO steel was investigated. The best magnetic properties $\left(B_{2500} \sim 1.85 \mathrm{~T}\right)$ were fixed for the samples with the lowest level of the initial magnetic induction and a copper content $\sim 0.5 \mathrm{wt} \%$ subjected to slow heating for PR and HTT and for copper-free samples after annealing for PR with high rate (without HTT).

In the copper-bearing material with the initial sharp texture, regardless of the heating rate, the nucleation of new grains following PR is connected with deformation twins; in samples without copper and with copper and diffuse texture, it is connected predominantly with shear bands and transition bands. It was found that, upon heating at a rate of $\sim 0.004 \mathrm{~K} / \mathrm{s}$, the PR temperature in copper-bearing samples is markedly higher than in the copper-free material. The mechanism of the analogous effect of copper on the structure upon deformation and recrystallization calls for further investigation. Upon heating for PR at a rate of $\sim 4 \mathrm{~K} / \mathrm{s}$, the formation of new grains occurs almost simultaneously for all of the studied samples. For all chemical compositions of alloys, the grain size in samples subjected to rapid heating for PR proved to be fairly close; yet, this size was substantially smaller than in samples heated with a rate of $\sim 0.004 \mathrm{~K} / \mathrm{s}$.

After HTT in copper-bearing samples with the initial sharp texture, regardless of the annealing rate for PR, the secondary-recrystallized grains occupy an appreciable part of the volume. After HTT in samples without copper and with copper and diffuse texture, anomalously grown crystallites are very rarely observed as individual grains.

Based on the experimental data, in order to obtain high final magnetic properties of the ultra-thin GOES produced by the Littmann method, it was suggested to use $\mathrm{Fe}-3 \% \mathrm{Si}-0.5 \% \mathrm{Cu}$ alloy with a diffuse orienta- tion of grains relative to the ideal orientation (110)[001] as the workpiece. When the ultra-thin GOES is produced from this material, the high-temperature refining annealing can be performed without secondary recrystallization or the degradation of the properties.

\section{ACKNOWLEDGMENTS}

This work was performed at the Laboratory "Methods of Structure Analysis and Properties of Materials and Nanomaterials" of the Center of Collaborative Access of the Ural Federal University.

\section{REFERENCES}

1. I. B. Kekalo and B. A. Samarin, Physical Metal Science of Precision Alloys. Alloys with Special Magnetic Properties (Metallurgiya, Moscow, 1989) [in Russian].

2. G. Xiuhua, Q. Kemin, and Q. Chunlin, "Magnetic properties of grain oriented ultra-thin silicon steel sheets processed by conventional rolling and cross shear rolling," Mater. Sci. Eng., A 430, 138-141 (2006).

3. N. H. Heo, J. Y. Soh, J. M. Oh, and S. B. Kim, "Influence of cold-rolling texture and heating rate on $\{110\}\langle 001\rangle$ development in inhibitor-free 3\% $\mathrm{Si}-\mathrm{Fe}$ sheets," J. Magn. Magn. Mater. 320, 635-637 (2008).

4. M. L. Lobanov, A. A. Redikul'tsev, G. M. Rusakov, I. V. Kagan, and O. V. Pervushina, "Effect of the grain orientation in the material used for the preparation of an ultrathin electrical steel on its texture and magnetic properties," Phys. Met. Metallogr. 111, 479-486 (2011).

5. V. A. Zaikova, I. E. Startseva, and B. N. Filippov, Domain Structure and Magnetic Properties of Electrotechnical Steels (Nauka, Moscow, 1992) [in Russian].

6. G. M. Rusakov, M. L. Lobanov, A. A. Redikul'tsev, and I. V. Kagan, "Specific features of cold deformation of a (110)[001] single crystal of an $\mathrm{Fe}-3 \% \mathrm{Si}-0.5 \% \mathrm{Cu}$ alloy related to twinning," Phys. Met. Metallogr. 111, 530-536 (2011).

7. M. L. Lobanov, G. M. Rusakov, A. A. Redikul'tsev, and I. V. Kagan, "Deformation-twinning-related features of primary recrystallization of (110) [001] single crystals of the $\mathrm{Fe}-3 \% \mathrm{Si}-0.5 \% \mathrm{Cu}$ alloy," Phys. Met. Metallogr. 111, 587-592 (2011).

8. B. K. Sokolov, A. K. Sbitnev, V. V. Gubernatorov, I. V. Gervasyeva, and L. R. Vladimirov, "On the influence of the annealing heating rate on the recrystallization texture of a deformed single crystal (110)[001] of 3\% silicon iron," Text. Microstruct. 26-27, 427-443 (1995).

Translated by I. Krasnov 